\title{
Chemical enhancer induced changes in the mechanisms of transdermal delivery of zinc oxide nanoparticles
}

\author{
Tsung-Rong Kuo ${ }^{\mathrm{a}}$, Chung-Long Wu ${ }^{\mathrm{a}}$, Chih-Ting Hsu ${ }^{\mathrm{b}}$, Wen Lo ${ }^{\mathrm{b}}$, Shu-Jen Chiang ${ }^{\mathrm{c}}$, Sung-Jan Lin ${ }^{\mathrm{d}, \mathrm{e}}$, \\ Chen-Yuan Dong ${ }^{\mathrm{b}, *}$, Chia-Chun Chen ${ }^{\mathrm{a}, \mathrm{c}, * *}$ \\ a Department of Chemistry, National Taiwan Normal University, Taipei 116, Taiwan \\ ${ }^{\mathrm{b}}$ Department of Physics, National Taiwan University, Taipei 106, Taiwan \\ ${ }^{\mathrm{c}}$ Institute of Atomic and Molecular Sciences, Academia Sinica, Taipei 106, Taiwan \\ ${ }^{\mathrm{d}}$ Institute of Biomedical Engineering, College of Medicine and College of Engineering, National Taiwan University, Taipei 106, Taiwan \\ e Department of Dermatology, National Taiwan University Hospital and College of Medicine, Taipei 106, Taiwan
}

\section{A R T I C L E I N F O}

\section{Article history:}

Received 22 November 2008

Accepted 2 February 2009

Available online 20 February 2009

\section{Keywords:}

Two-photon microscopy

Second harmonic generation

ZnO nanoparticles

Chemical enhancer

Transdermal

\begin{abstract}
A B S T R A C T
The overlapping wavelength of photoluminescence ( $\mathrm{PL}$ ) of zinc oxide nanoparticles ( $\mathrm{ZnO} \mathrm{NPs}$ ) and autofluorescence (AF) from the stratum corneum (SC) has for a long time held back researchers from investigating the chemically enhanced penetration pathways of ZnO NPs into the SC lipids. However, the non-linear polarization effect of second harmonic generation (SHG) may be used for ZnO NPs to be distinguished from the AF of the SC. This study combined the SHG of ZnO NPs and the AF of the SC to image the transdermal delivery of ZnO NPs under the chemical enhancer conditions of oleic acid (OA), ethanol $(\mathrm{EtOH})$ and oleic acid-ethanol $(\mathrm{OA}-\mathrm{EtOH})$. In addition to qualitative imaging, the microtransport properties of $\mathrm{ZnO}$ NPs were quantified to give the enhancements of the vehicle-to-skin partition coefficient $(K)$, the SHG intensity gradient $(G)$ and the effective diffusion path length $(L)$. The results showed that $\mathrm{OA}, \mathrm{EtOH}$ and $\mathrm{OA}-\mathrm{EtOH}$ were all capable of enhancing the transdermal delivery of ZnO NPs by increasing the intercellular lipid fluidity or extracting lipids from the SC.
\end{abstract}

(c) 2009 Elsevier Ltd. All rights reserved.

\section{Introduction}

At the present, one of the fastest growing scientific fields is that of nanoscale discovery and application. Of particular concern are the uses of nanoparticles (NPs) in applications ranging from targeted fluorescent labels in the life sciences [1], bacterial inhibitors [2], and ultraviolet radiation (UVR) protective cosmetics [3]. Specifically, inorganic ZnO NPs are popular active substances used in UVR protective substances due to their broad spectral absorption in the UVA range [4]. Overexposure of human skin to UVR may lead to sunburn and an increased risk of skin cancer [5-7]. To reduce skin damages caused by UVR, many health care practitioners have advocated UVR protective ingredients for a wide variety of dermatological products $[8,9]$. Application of UVR protective ingredients on top of the skin is indicated to penetrate ZnO NPs into

\footnotetext{
* Corresponding author.

** Corresponding author. National Taiwan Normal University, Department of Chemistry, 88 Sec. 4, Ting Chow Road, Taipei 116 Taiwan, Taipei 116, Taiwan. Fax: +886289316363.

E-mail addresses: cydong@phys.ntu.edu.tw (C.-Y. Dong), cjchen@ntnu.edu.tw (C.-C. Chen).
}

the SC of the skin. Based on inherent barrier properties, the SC inhibits transdermal delivery of hydrophilic and large molecular weight molecules [10,11]. The plasma membrane of the cell is mainly composed of various types of phospholipids and membrane proteins. The membrane proteins transport hydrophilic and larger uncharged polar molecules (such as glucose and ions) across membrane. However, organic compounds such as OA and EtOH are chemical enhancers that can alter the packing structure of the SC lipids to increase lipid fluidity and reduce skin resistance [12,13]. A better understanding of the transdermal delivery of ZnO NPs via chemically enhanced pathways may optimize the applications and address biocompatibility issues of NPs in the SC.

Recently, multi-photon microscopy has been used as a noninvasive method for the direct visualization of skin structures [14,15]. In multi-photon imaging, fluorophore excitation is achieved by the non-linear absorption process $[16,17]$. Specifically, in twophoton microscopy, two near-infrared (near-IR) photons of approximately half the one-photon excitation energy are simultaneously absorbed by the fluorescent molecule $[18,19]$. This approach can be used to excite intrinsic or extrinsic fluorophores, which are generally excited by ultraviolet (UV) or visible (VIS) light [20]. The near-IR radiation allows deeper penetration into the 
imaged tissues and the point-like excitation volume greatly can reduce overall specimen photodamage [21]. For skin imaging, down to tissue depths of $100-200 \mu \mathrm{m}$, the AF of human skin can be detected [22,23]. For transdermal delivery studies, two-photon microscopy has provided three-dimensional (3-D) images detailing the chemical enhancer- and ultrasound-induced changes in the distributions of fluorescent probes across human skin [24-26].

Despite of the advances in the study of transdermal transport of molecular dyes, the fluorescence of ZnO NPs and SC were almost overlapped that held back researchers from investigating the transdermal delivery of ZnO NPs with or without enhancers. To tackle this issue, the current study examined the non-linear polarization effect of SHG for ZnO NPs, based on its unique polarizable property as well as non-centrosymmetric structure $[27,28]$. The generated secondharmonic signal at twice the frequency (half the wavelength) of the excitation source was observed in ZnO NPs. After confirmation of the SC with no SHG, the combination of the SHG of ZnO NPs and AF of SC was used to image and characterize the penetration pathway and depth of ZnO NPs into nude mouse skin under three chemical enhancer conditions: $\mathrm{OA}, \mathrm{EtOH}$, and $\mathrm{OA}-\mathrm{EtOH}$. In addition to qualitative imaging, the microtransport properties of the ZnO NPs, including the $K, G$ and $L$, were quantified relative to the control sample (without chemical enhancers).

\section{Materials and methods}

\subsection{Preparation of $\mathrm{ZnO}$ nanoparticles}

ZnO NPs were prepared by adding $5.280 \mathrm{~g}$ of zinc acetate dehydrate (reagent grade, Sigma-Aldrich) in $250 \mathrm{~mL}$ EtOH (99.5\%, Showa) and the solution was heated until it become clear [29]. After the solution was refluxed for $1 \mathrm{~h}$ at $78^{\circ} \mathrm{C}$, approximate $60 \%$ of the solvent was removed by distillation and replaced by the same amount of fresh EtOH. The solution was then placed in ice bath at $0{ }^{\circ} \mathrm{C}$ and $1.392 \mathrm{~g}$ of lithium hydroxide (98+\%, Sigma-Aldrich) was added under ultrasonication until a transparent solution was obtained. After $60 \mathrm{~min}$ of reaction time, the solution consisting of small ZnO NPs with around $2 \mathrm{~nm}$ diameter was filtered through a $0.1 \mu \mathrm{m}$ glass fiber filter to remove undissolved $\mathrm{LiOH}$.

To avoid particle aggregation, $\mathrm{ZnO}$ NPs with a diameter of approximate $10 \mathrm{~nm}$ was achieved by continuous growth of $2 \mathrm{nmZnONPs}$. The solution containing $2 \mathrm{~nm}$ particles was heated and mixed with $10 \mathrm{~mL}$ of deionized water at $60{ }^{\circ} \mathrm{C}$ for about $6 \mathrm{~min}$. During the cooling process, white precipitate was formed within the solution. The precipitate was separated from the supernatant solution by centrifugation at $3800 \mathrm{rpm}$ for about $10 \mathrm{~min}$. Next, the precipitate was washed with an EtOH-deionized water solution (19:1 by volume) and centrifuged again. The last two steps were repeated at least four times to ensure the removal of physisorbed ionic compounds. The obtained ZnO NPs have mainly the same diameter of approximate $10 \mathrm{~nm}$, and are spherical in shape. The absorption and emission peaks of ZnO NPs are located at $370 \mathrm{~nm}$ and $525 \mathrm{~nm}$, respectively.

\subsection{Donor solution preparation}

The donor vehicle solutions of ZnO NPs were consisted of EtOH, OA, and PBS (99\%, Sigma). EtOH, OA, PBS and ZnO NPs with different weight ratio were prepared
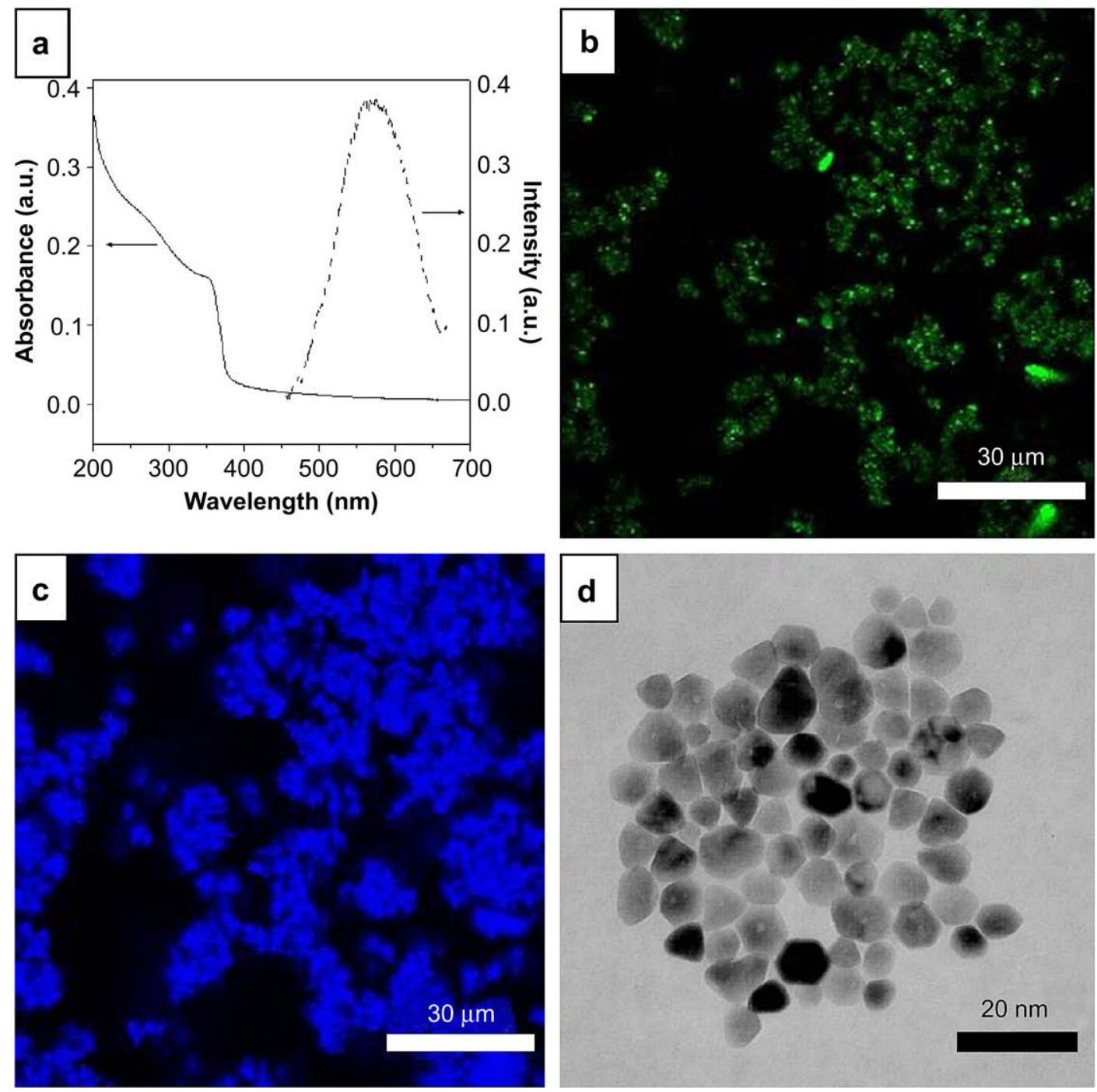

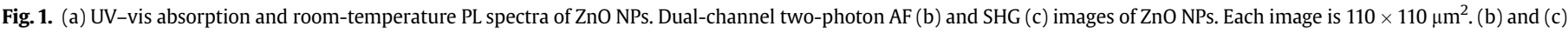
The two-photon AF (green) and SHG (blue) of ZnO NPs, respectively. (d) The TEM image of ZnO NPs with spherical shape. 
for the chemical enhancer experiments. EtOH and OA were used as the chemical enhancer in comparison with control solution of PBS. In the control donor solution, $10 \%(w / w)$ of $\mathrm{ZnO}$ NPs was added to the PBS solution. The OA donor solution was prepared by adding $10 \%$ of ZnO NPs and $5 \%$ of OA $(w / w)$ in PBS solution. The EtOH donor solution is consisted of $10 \%$ of $\mathrm{ZnO} \mathrm{NPs}(\mathrm{w} / \mathrm{w})$ and $45 \%$ of ethanol $(\mathrm{w} / \mathrm{w})$ in PBS solution. Finally, the OA-EtOH donor solution is composed of $10 \%$ of ZnO NPs, $5 \%$ of $\mathrm{OA}$ and $45 \%$ of $\mathrm{EtOH}(\mathrm{w} / \mathrm{w})$ in PBS solution.

\subsection{Preparation of the skin samples of nude mice for multi-photon microscopy imaging}

The skin specimens for the $\mathrm{ZnO}$ NPs diffusion experiments were obtained from the dorsal region of four 10-month-old nude mice (BALB/cAnN.Cg-Foxnlnu/CrlNarl), purchased from Laboratory Animal Center, NLAC. After the subcutaneous fat of the skin was removed using a knife, the skin specimens were then sectioned and mounted in vertical Franz diffusion cells. The acceptor chambers of the Franz diffusion cells were filled with PBS and the donor chambers were filled with $400 \mu \mathrm{L}$ of donor solution. After $12 \mathrm{~h}$, the skin samples were removed from Franz diffusion cells and excess formulation on the skin surface was washed 3-4 times with PBS. The circular region of the skin exposed to the donor solution was removed using a knife and then placed on a microscope slide. The skin specimen was then sealed with a glass coverslip for viewing. Note that the conditions of steady state of ZnO NPs diffusion were verified in calculating the parameters of ZnO NPs penetration. The SHG intensity profiles were measured for the diffusion times of 10 and $12 \mathrm{~h}$. It was found that the gradient profiles of SHG intensity of ZnO NPs were similar under the same donor solution condition. In this study, the diffusion time of $12 \mathrm{~h}$ was selected for the experiment.

\subsection{Dual-channel multi-photon microscopy}

A home-built two-photon microscope based on a commercial upright microscope (E800; Nikon, Japan) was used in this study. The output of a titanium-sapphire pulse laser (Tsunami TM, Spectra Physics, Mountain View CA) pumped by a diode-pumped, solid state laser (Millennia TM X, Spectra Physics, Mountain View, CA) operating at the wavelength of $780 \mathrm{~nm}$ was used as the excitation source. Upon reflection by the short-pass, main dichroic mirror (700dcspruv-3p, Chroma Technology, Rockingham, VT), circularly polarized laser source was then focused by an oil-immersion objective (S Fluor, NA 1.3; Nikon, Melville, NY). After the signals were generated, the broadband AF and SHG were separated by a secondary dichroic mirror (435 dcxr, Chroma Technology) and filtered respectively by two band-pass filters (MF: E435lp-700sp, SHG: HQ390/ 20, Chroma Technology) for the respective detection of broadband AF $(435-700 \mathrm{~nm})$ and SHG signals (380-400 nm). Single-photon counting photomultiplier tubes (R7400P, Hamamatsu, Japan) were used in this system. Each optical scan was composed of $512 \times 512$ pixels. For large-area scans of the skin specimens, a two-dimensional stage scanning system (H101; Prior Scientific Instruments, Cambridge, UK) was used for specimen translation, after each $x-y$ scan of the sample.

\subsection{Data collection and analysis}

Image software (National Institutes of Health, Bethesda, MD) was utilized to process images. For each skin sample, four sites were examined and each of the sites was $330 \times 330 \mu \mathrm{m}^{2}$ in size. The $z=0 \mu \mathrm{m}$ position was defined by the imaging depth where the highest average SHG count was detected. At each depth, ImageJ averages the SHG counts associated with the pixels in the 512 by 512 pixel image of the imaged field. For each site analyzed by ImageJ, a plot of the average SHG count versus the corresponding skin depth was used to generate the site-specific average SHG spatial distribution profile (SSDP).

The values for $E_{K}, E_{G}$ and $E_{L}$, were obtained following previously published methods [22-24]. The $E_{K}$ value was evaluated from the average SHG intensity on the surface of each of the four sites of sample skins. Assuming that the SHG intensity of the $\mathrm{ZnO}$ NPs permeant, $I$, is directly proportional to its concentration in the skin, $E_{K}$

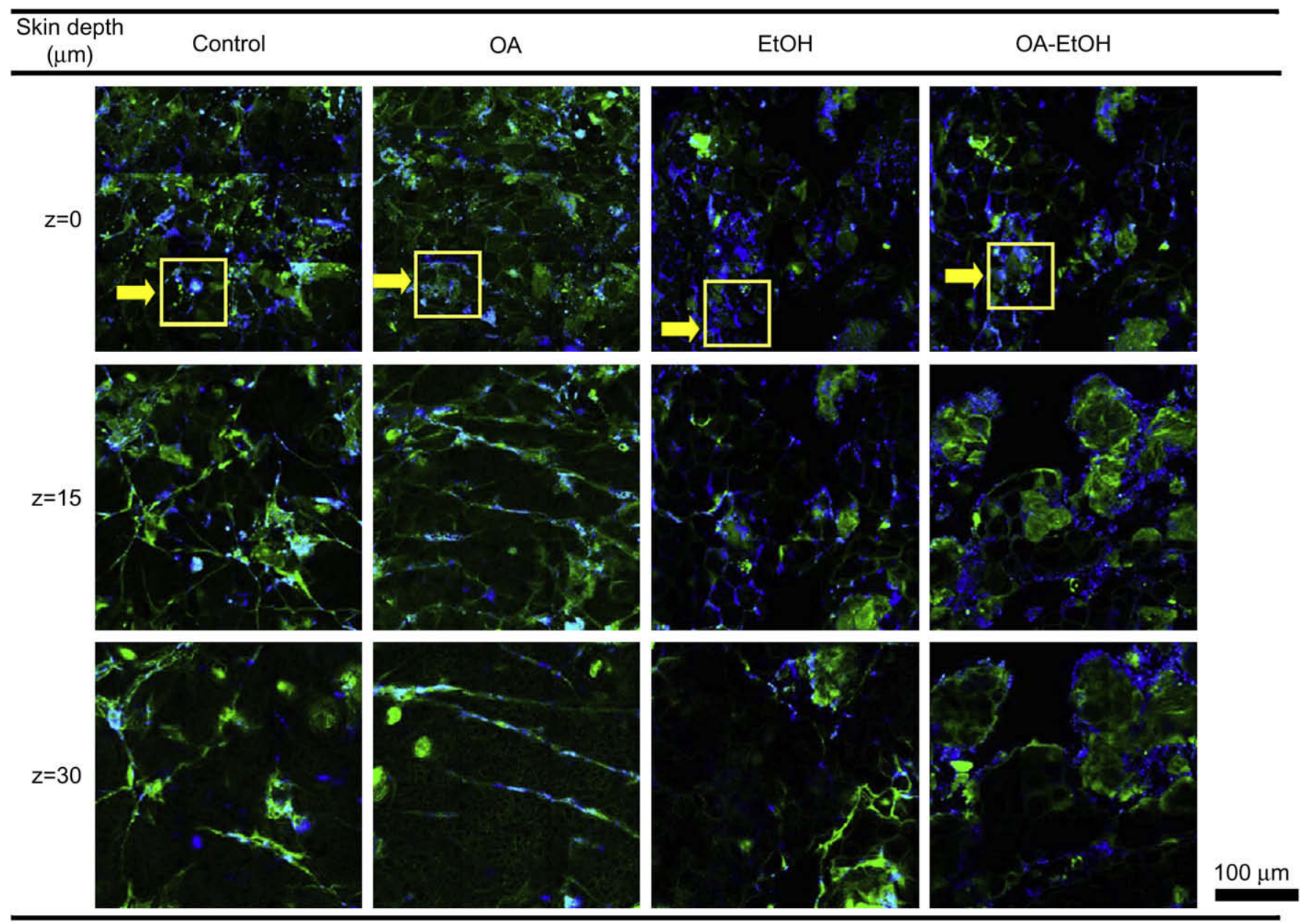

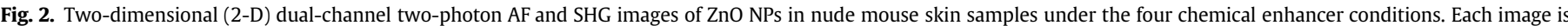

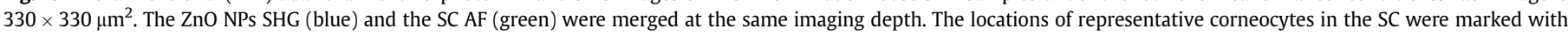
a yellow arrow. 
Table 1

The weight ratios of the four donor vehicle solutions consisting of EtOH, OA, OAEtOH and PBS

\begin{tabular}{lllll}
\hline Donor solution & ZnO NPs & PBS & Oleic acid & Ethanol \\
\hline Control & 1 & 9 & - & - \\
OA & 1 & 8.5 & 0.5 & - \\
EtOH & 1 & 4.5 & - & 4.5 \\
OA-EtOH & 1 & 4 & 0.5 & 4.5 \\
\hline
\end{tabular}

can be approximated from the average SHG intensity profiles using the following equation:

$E_{K}=\frac{I_{\text {Enhancer }}(z=0)}{I_{\text {Control }}(z=0)}$

where $I_{\text {Enhancer }}(z=0)$ is the average value of the SHG intensity of ZnO NPs on the surface of the chemically enhanced skin samples and $I_{\text {Control }}(z=0)$ is the average value of the SHG intensity of $\mathrm{ZnO}$ NPs on the surface of the control (PBS) skin samples.

The SHG intensity gradient, $G$, in the SC was evaluated based on the average intensity values and calculated by performing a linear regression for the data points comprising the first 11 axial scans of the skin. In calculating the enhancement in the $\mathrm{ZnO}$ NPs permeant intensity gradient, $E_{G}$, the following equation was used:

$E_{G}=\frac{(d I / d z)_{\text {Enhancer }}}{(d I / d z)_{\text {Control }}}$
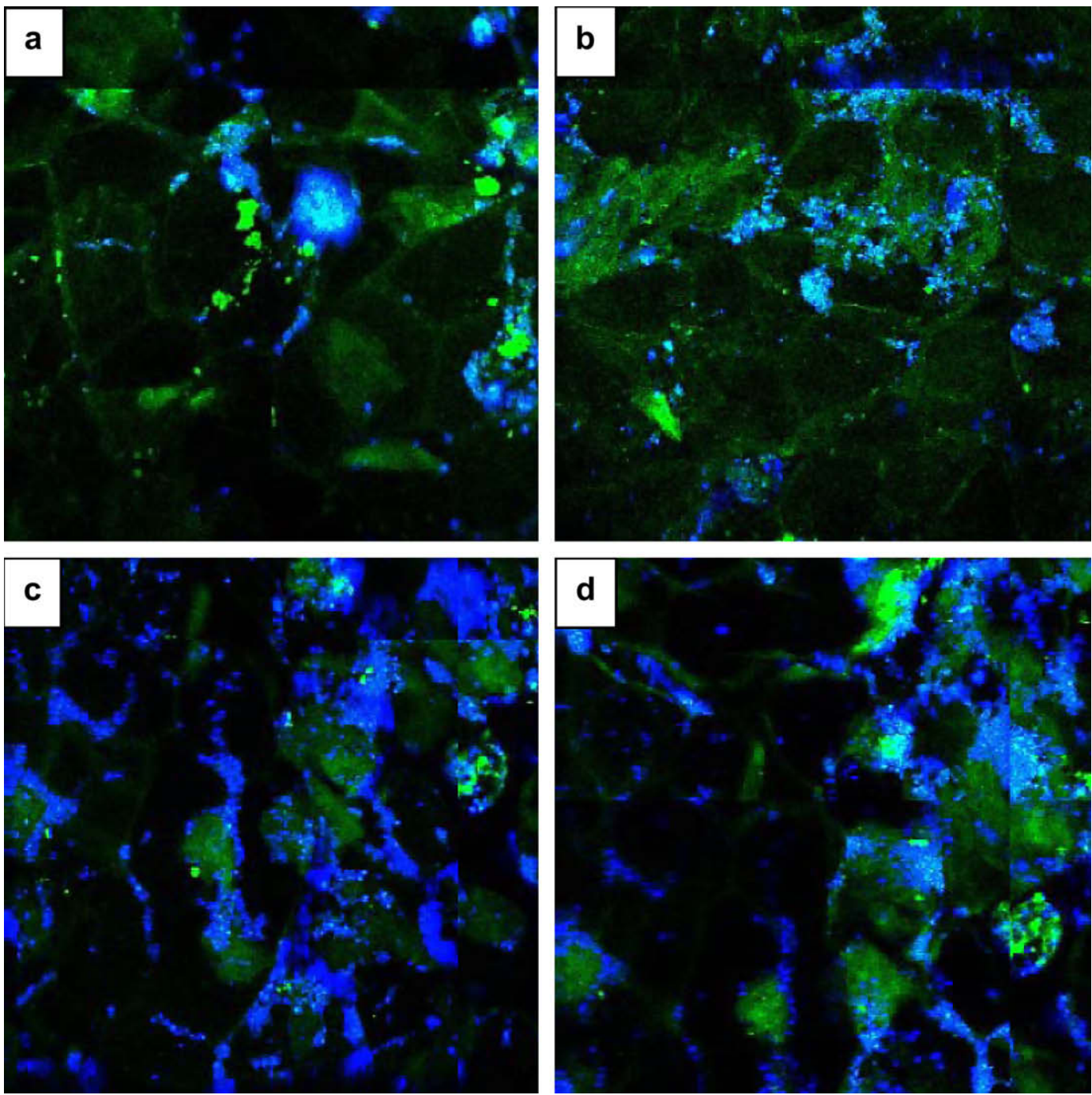

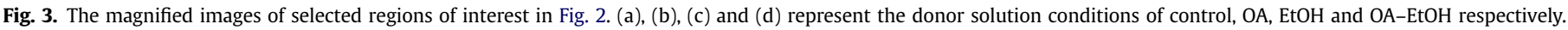


the size $\left(110 \times 100 \mu \mathrm{m}^{2}\right)$ of ZnO NPs. Displayed in Fig. $1 \mathrm{~b}$ and $\mathrm{c}$ are, respectively, the two-photon AF (bandwidth 435 to $700 \mathrm{~nm}$ ) and SHG (bandwidth $380-400 \mathrm{~nm}$ ) images of ZnO NPs. The dualchannel two-photon images show that ZnO NPs can be effectively imaged by two-photon microscopy. Besides the optical images, the ZnO NPs were also characterized by transmission electron microscopy (TEM). The electron microscopic image (Fig. 1d) indicated that the averaged diameter of the NPs was $\sim 10 \mathrm{~nm}$.

\subsection{Imaging the chemical enhanced transdermal delivery of ZnO NPs}

To analyze the transdermal transport of ZnO NPs with different chemical enhancers, the representative dual-channel two-photon images of $\mathrm{ZnO}$ NPs distribution in the nude mouse SC are shown in Fig. 2, while the formulations of four donor solutions, control and enhancers $\mathrm{OA}$, EtOH and $\mathrm{OA}-\mathrm{EtOH}$, are presented in Table 1. In Fig. 2, the SHG images (blue pseudocolor) show the distributions of ZnO NPs and AF (green pseudocolor) is used to indicate the SC of the skin. Where appropriate, the locations of representative corneocytes in the SC are marked with a yellow arrow in each image, as the corneocytes can be clearly identified. Moreover, Fig. 3 shows the magnified images of selected regions of interest as marked in Fig. 2. The SHG of ZnO NPs and the AF of SC are merged at the same imaging depth. The images are presented in column at $z=0 \mu \mathrm{m}$
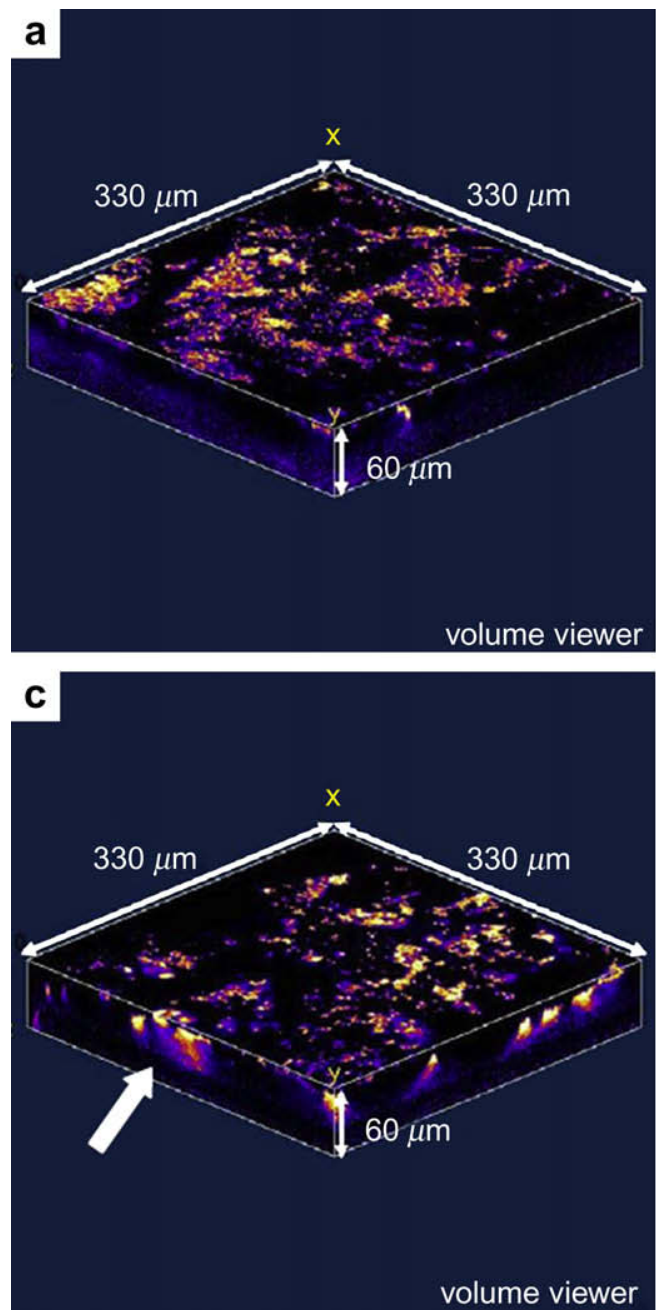

(the skin surface), $z=15 \mu \mathrm{m}$ and $z=30 \mu \mathrm{m}$, to provide qualitative information on the amount and locations of ZnO NPs with chemical enhancers.

From the dual-channel two-photon images, the ZnO NPs are observed to transport between the intercellular domains of the SC and distribute heterogeneously within the SC. In addition, the majority of the second harmonic generation of ZnO NPs is found to locate at the uppermost layers of the skin at approximate depths around $30 \mu \mathrm{m}$, with insignificant SHG detected beyond that depth. The result suggests that the SC acts as the primary barrier to the transdermal transport of ZnO NPs and it is through the multilamellar lipid regions between the corneocytes that the primary $\mathrm{ZnO}$ NPs transport across the mouse skins. Furthermore, the tendency of ZnO NPs to distribute in the intercellular domains suggests that the intracellular pathways are not effective for $\mathrm{ZnO}$ NPs delivery. These suggestions correspond to the accumulation of ZnO NPs on the surface of human skin and around corneocytes presented by electron micrographs [33].

\subsection{3-D SHG images of ZnO NPs distribution in the SC under different chemical enhancer conditions}

To illustrate the extent of ZnO NPs penetration, axial-scans using SHG microscopy was applied to show the 3-D distribution of $\mathrm{ZnO}$
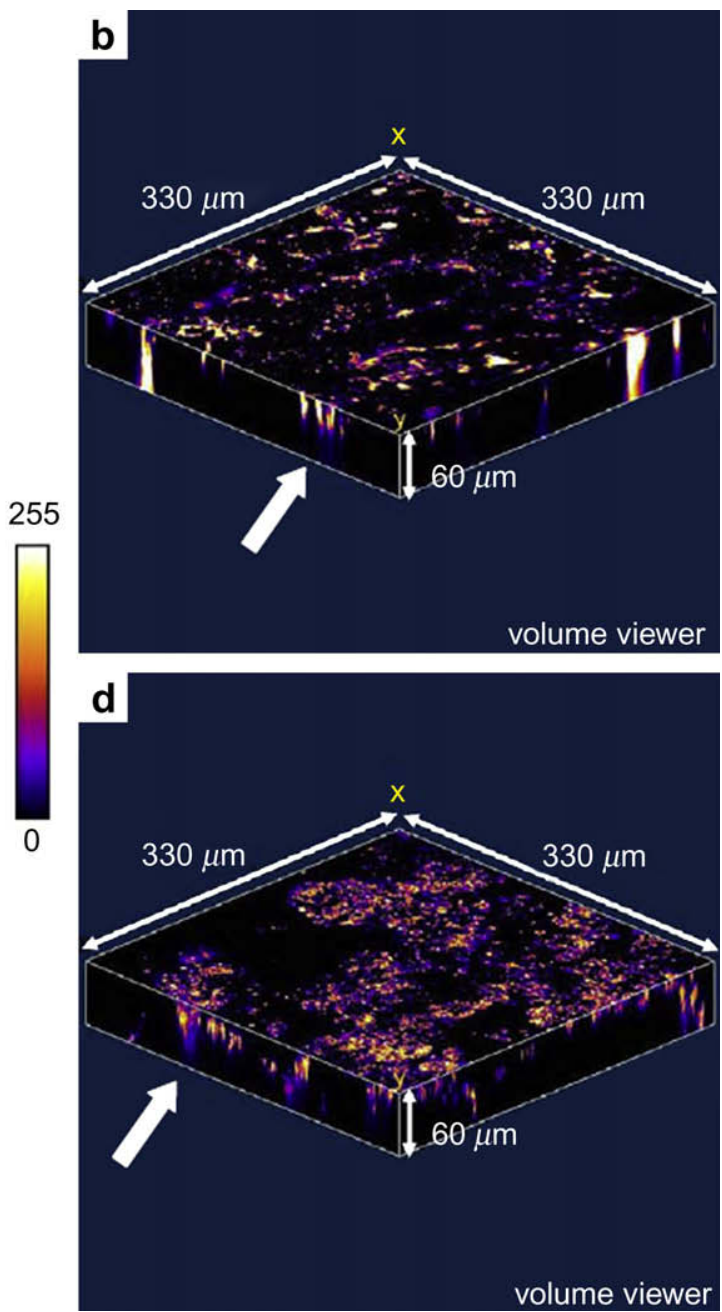

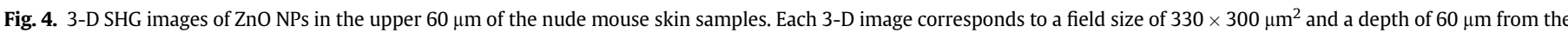

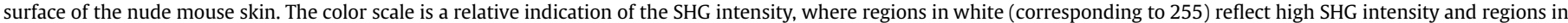

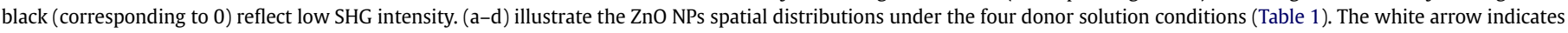
the penetration pathway of $\mathrm{ZnO}$ NPs into the SC. 
NPs. In Fig. 4, each 3-D image stack represents a field of view $330 \times 300 \mu \mathrm{m}^{2}$ in size and at a depth of $60 \mu \mathrm{m}$ from the surface of the skin. Fig. 4a-d illustrates the SHG spatial distributions of $\mathrm{ZnO}$ NPs across the skin sample $(60 \mu \mathrm{m})$ with four donor solutions, control and enhancers OA, EtOH and OA-EtOH, respectively. The attached color scales were used for these images.

The penetration pathways of ZnO NPs are visible in the 3-D images of Fig. 4. A significant quantity of $\mathrm{ZnO}$ NPs accumulation indicated by white arrows reveals the transdermal pathway of $\mathrm{ZnO}$ NPs with chemical enhancers. Such result correlates with the previous discussion that ZnO NPs with chemical enhancers transport through intercellular pathway to penetrate into SC and do not enter the corneocytes. Moreover, the axial distribution of ZnO NPs with OA enhancer appears to increase at channel-like area, compared with control solution. The increase in axial distribution reflects the increased intensity of SHG signals. For EtOH enhancer, the SHG signal intensity of ZnO NPs is apparently spread out across the SC. The essence of the axial distribution for ZnO NPs with $\mathrm{OA}-\mathrm{EtOH}$ enhancer is closer to the axial distribution of ZnO NPs with EtOH enhancer, rather than that of ZnO NPs with OA enhancer.

\subsection{Axial SHG intensity profiles of ZnO NPS}

In addition to qualitative imaging, we also determined the average $\mathrm{ZnO}$ SHG intensity profiles under different chemical enhancer conditions and the results are shown in Fig. 5. The average SHG intensity of each donor solution was plotted on the $y$ axis and the depth $(z)$ from the skin surface (at $z=0 \mu \mathrm{m}$ ) was plotted on the $x$ axis in $\mu \mathrm{m}$ scale. The error bars represent standard deviation (SD) of the average value of the ZnO NPs SHG intensity.

From the profiles, the SHG intensities of $\mathrm{ZnO}$ NPs with OA, EtOH and $\mathrm{OA}-\mathrm{EtOH}$ enhancers on the skin surface appear to be higher than that of ZnO NPs with control donor solution. As the depth of the skin increases, the SHG signals of ZnO NPs with OA enhancer become to decay slower than those of ZnO NPs with the individual EtOH and OA-EtOH enhancers. In regard to EtOH and OA-EtOH enhancers, the increases in the SHG intensity gradient are almost the same. With noise signals defined by less than one-tenth of the SHG intensity, the penetration depths of ZnO NPs with control, enhancers OA, EtOH and OA-EtOH were 10, 30, 25 and $20 \mu \mathrm{m}$ from the skin surface.

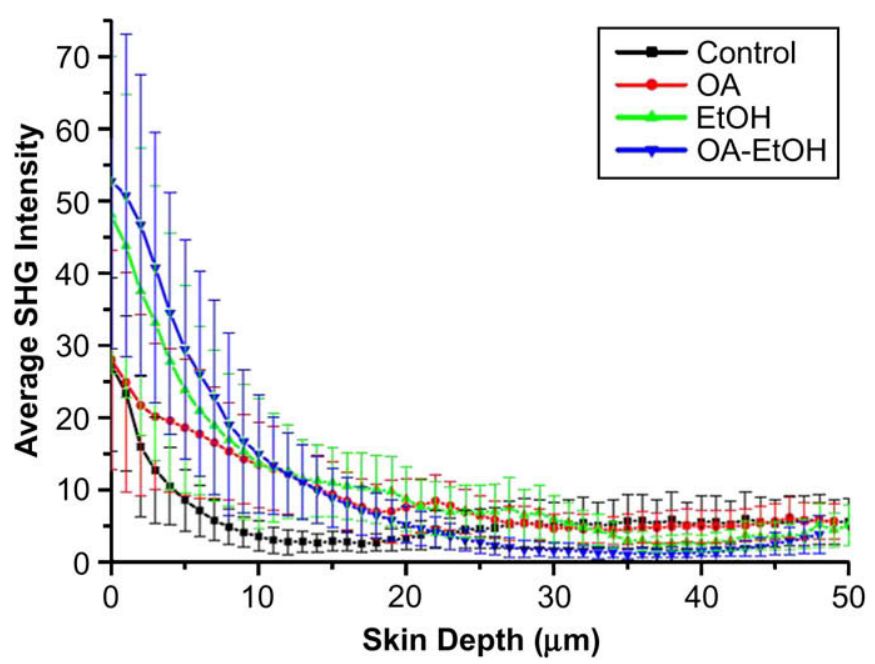

Fig. 5. Axial ( $z$ ) SHG intensity profiles of ZnO NPs in nude mouse skins (skin surface at $z=0 \mu \mathrm{m}$ ). The average SHG intensity profiles of ZnO NPs under the four chemical enhancer conditions (Table 1) are shown.
3.5. Chemical enhancements in the vehicle-to-skin partition coefficient, the intensity gradient and the effective diffusion path length for $\mathrm{ZnO}$ NPS

To quantify our imaging results, we calculated from the average SHG intensity profiles in Fig. 5 and listed, in Table 2, the $E_{K}$ values of the $\mathrm{ZnO} N$ Ns at the skin surface, $I(z=0)$, for enhancers OA, EtOH and $\mathrm{OA}-\mathrm{EtOH}$, respectively. To calculate the $E_{G}$, values, the intensity gradients were determined from the average SHG intensity profiles and the results are $-3.26 \pm 1.73,-2.02 \pm 1.36,-4.74 \pm 3.04$ and $-4.81 \pm 3.67$, for control, enhancers OA, EtOH and OA-EtOH respectively. Linear regression yields $R^{2}$ values of control, enhancers OA, EtOH and OA-EtOH to be 0.92, 0.94, 0.99 and 0.99, respectively. Based on the slope values determined, the $E_{G}$ values for enhancers OA, EtOH and OA-EtOH are $0.62 \pm 0.86,1.45 \pm 0.83$ and $1.48 \pm 0.93$, respectively. Utilizing the equation $E_{L}=E_{K} / E_{G}$, the values of enhancers $\mathrm{OA}$, EtOH and OA-EtOH are $1.85 \pm 1.44$, $1.25 \pm 0.65$ and $1.30 \pm 0.93$, respectively. For reference, the enhancement values calculated for sulforhodamine B (SRB) with oleic acid enhancer are: $E_{K}=4.56 \pm 2.05, E_{L}=3.66 \pm 1.67$, and $E_{G}=1.24 \pm 0.09$ [25].

From the $E_{K}$ value of $1.15 \pm 0.46$ for OA enhancer, the accumulation of ZnO NPs on the skin surface is slightly increased by $15 \%$ OA is capable of altering SC lipid ordering to change the transdermal permeability of the permeant. The increase of ZnO NPs on the skin surface can thus be explained by the formation of separate domains of OA pools within the SC lipids. Such explanation corresponds to $E_{G}=0.62 \pm 0.86$ for OA enhancer. $E_{G}(0.62 \pm 0.86)<1$ reflects the fact that the delivery of ZnO NPs into SC is through the phaseseparated OA domains. After ZnO NPs accumulate into OA pools, the decreasing rate of the SHG intensity gradient is $62 \%$ for OA enhancer, compared with the control. The observed increase in $E_{L}=1.85 \pm 1.44$ for OA enhancer verifies the creation of a microtransport environment in the SC.

For EtOH enhancer, the $E_{K}$ value of $1.81 \pm 0.56$ shows that chemical enhancer of EtOH can enhance the accumulation of $\mathrm{ZnO}$ NPs on the skin surface by $81 \%$. EtOH is a potent solvent for both polar and non-polar species. Therefore, it is possible that the presence of $\mathrm{EtOH}$ in the donor solution is capable of leaching significant quantities of non-covalently bound amphiphilic SC lipids, causing the modulation of the skin barrier. However, the enhancement of $E_{G}$ value for EtOH enhancer is $1.45 \pm 0.83>1$, indicating that the decreasing rate of the SHG intensity gradient is faster than control by $145 \%$. Therefore, the effect of the modulation of skin barrier caused by EtOH enhancer is constrained, allowing $\mathrm{ZnO}$ NPs to accumulate merely on the skin surface with a minimum penetration. The $E_{L}$ value of the EtOH enhancer, $1.25 \pm 0.65$, reflects the accumulation of ZnO NPs on the skin surface associated with the fast decreasing rate of the SHG intensity gradient.

As regard to OA-EtOH enhancer, the $E_{K}$ value of $1.93 \pm 0.62$ demonstrates the significant $\mathrm{ZnO}$ accumulation on the skin surface by $93 \%$. Compared with EtOH donor solution, the additional presence of OA in OA-EtOH donor solution appears to increase the $\mathrm{ZnO}$ NPs accumulation by only $12 \%$. Whereas, compared to OA solution, adding EtOH enhancer to OA donor solution, the accumulation of ZnO NPs increases less than $81 \%$. This phenomenon suggests the

Table 2

Calculated values of $E_{K}, E_{G}$, and $E_{L}$, for transdermal delivery of ZnO NPs.

\begin{tabular}{llllll}
\hline Donor solution & $I(z=0)$ & $d I / d z(g)$ & $E_{K}$ & \multicolumn{1}{l}{$E_{G}$} & $E_{L}$ \\
\hline Control & $31.78 \pm 13.86$ & $-3.26 \pm 1.73$ & - & - & - \\
OA & $35.44 \pm 5.80$ & $-2.02 \pm 1.36$ & $1.15 \pm 0.46$ & $0.62 \pm 0.86$ & $1.85 \pm 1.44$ \\
EtOH & $57.42 \pm 20.15$ & $-4.74 \pm 3.04$ & $1.81 \pm 0.56$ & $1.45 \pm 0.83$ & $1.25 \pm 0.65$ \\
OA-EtOH & $61.31 \pm 27.15$ & $4.81 \pm 3.67$ & $1.93 \pm 0.62$ & $1.48 \pm 0.93$ & $1.30 \pm 0.71$ \\
\hline
\end{tabular}


mutual interference of OA and EtOH effects on the SC lipids. The phase-separated domains formed by OA enhancer to decrease the skin barrier are disturbed by the effect of EtOH enhancer in modulating the SC lipid fluidity. The decreasing rate of the SHG intensity gradient for OA-EtOH enhancer is shown by the $E_{G}$ value of $1.48 \pm 0.93>1$, with $86 \%$ faster than OA enhancer and with $3 \%$ faster than EtOH enhancer. Consequently, the $E_{L}$ value of $1.30 \pm 0.71$ for OA-EtOH enhancer indicates that the SC lipid fluidity increased by $\mathrm{OA}$ enhancer is offset by the leaching effect of EtOH enhancer.

\section{Conclusion}

In this work, we succeed in combining the second harmonic generation of $\mathrm{ZnO}$ nanoparticles and autofluorescence of the stratum corneum to image the transdermal pathway of $\mathrm{ZnO}$ nanoparticles with oleic acid, ethanol and oleic acid-ethanol enhancers, using two-photon microscopy. To quantify dual-channel two-photon microscopy images, the enhancement values of vehicle-to-skin partition coefficient and second harmonic generation intensity gradient were calculated from the second harmonic generation signal intensity of $\mathrm{ZnO}$ nanoparticles. The ratio of the enhancement values of vehicle-to-skin partition coefficient and second harmonic generation intensity gradient reflects the enhancement values of effective diffusion path length. The results suggested that the multilamellar lipid regions between the corneocytes were the pathways for $\mathrm{ZnO}$ nanoparticles delivery. With oleic acid enhancer, the transport of $\mathrm{ZnO}$ nanoparticles into the stratum corneum is endorsed by the phase-separated oleic acid domains, while ethanol enhancer leaches significant amount of non-covalently bound amphiphilic stratum corneum lipids to modulate the skin barrier. As regards oleic acid-ethanol donor solution, the increase of stratum corneum lipid fluidity associated with oleic acid enhancer was offset by the effect of ethanol in loosening the stratum corneum lipid structure. Among our choices of the different chemical enhancer conditions, the oleic acidethanol combination can be regarded as the most effective donor solution in transdermal $\mathrm{ZnO}$ nanoparticles into the stratum corneum.

\section{Acknowledgments}

This study was financially supported by the National Science Council (NSC), Taiwan (NSC-96-3112-B-002-025, NSC-95-3112-B002-019, NSC-94-3112-B-002015Y, and NSC-96-2120-M003-001), and National Taiwan University Hospital (975-842). The twophoton experiments and data analysis were performed at the Optical Molecular Imaging Microscopy Core Facility (A5) of the National Research Program for Genomic Medicine of NSC in Taiwan.

\section{Appendix}

Figures with essential color discrimination. Certain images in the majority of figures in this article are difficult to interpret in black and white. The full color images can be found in the on-line version, at doi:10.1016/j.biomaterials.2009.02.003.

\section{References}

[1] Akermann ME, Chan WCW, Laakkonen P, Bhatia SN, Ruoslahti E. Nanocrystal targeting in vivo. Proc Natl Acad Sci U S A 2002;99:12617-21.

[2] Chen X, Schluesener HJ. Nanosilver: a nanoproduct in medical application. Toxicol Lett 2008;176:1-12.
[3] Serpone N, Emeline AV. Modelling heterogeneous photocatalysis by metaloxide nanostructured semiconductor and insulator materials: factors that affect the activity and selectivity of photocatalysts. Res Chem Intermed 2005;31:391-432.

[4] Diffey BL, Grice J. The influence of sunscreen type on photoprotection. J Invest Dermatol 1997;137:103-5.

[5] Sachsenmaier C, Radler-Pohl A, Zinck R, Nordheim A, Herrlich P, Rahmsdorf H. Involvement of growth factor receptors in the mammalian UVC response. Cell 1994;78:963-72.

[6] Roberts LK, Beasley DG. Commercial sunscreen lotions prevent ultravioletradiation-induced immune suppression of contact hypersensitivity. J Invest Dermatol 1995;105:339-44.

[7] Rosette C, Karin M. Ultraviolet light and osmotic stress: activation of the JNK cascade through multiple growth factor and cytokine receptors. Science 1996;274:1194-7.

[8] Lavker R, Kaidbey K. The spectral dependence for UVA-induced cumulative damage in human skin. J Invest Dermatol 1997;108:17-21.

[9] Choquenet B, Couteau C, Paparis E, Coiffard JM. Quercetin and rutin as potential sunscreen agents: determination of efficacy by an in vitro method. J Nat Prod 2008;71:1117-8.

[10] Rouse JG, Yang J, Ryman-Rasmussen JP, Barron AR, Monteiro-Riviere NA. Effect of mechanical flexion on the penetration of fullerene amino acid-derivatized peptide nanoparticles through skin. Nano Lett 2007;7:155-60.

[11] Mortensen LJ, Oberdorster G, Pentland AP, DeLouise LA. In vivo skin penetration of quantum dot nanoparticles in the murine model: the effect of UVR. Nano Lett 2008;8:2779-87.

[12] Heard CP, Kung D, Thomas CP. Skin penetration enhancement of mefenamic acid by ethanol and 1,8-cineole can be explained by the 'pull' effect. Int J Pharm 2006;321:167-70.

[13] Pierre M, Tedesco AC, Bentley M. Oleic acid as optimizer of the skin delivery of 5-aminolevulinic acid in photodynamic therapy. Pharm Res 2006;23:360-6.

[14] Masters BR, So PT, Gratton E. Multiphoton excitation fluorescence microscopy and spectroscopy of in vivo human skin. Biophys J 1997;72:2405-12.

[15] Masters BR, So PT. Confocal microscopy and multi-photon excitation microscopy of human skin in vivo. Opt Express 2001;1:2-10.

[16] Svoboda K, Denk W, Kleinfeld D, Tank DW. In vivo dendritic calcium dynamics in neocortical pyramidal neurons. Nature 1997;385:161-5.

[17] Gerritsen HC, Grauw CJ. Imaging of optically thick specimen using two-photon excitation microscopy. Microsc Res Tech 1999;47:206-9.

[18] Diaspro A. Introduction to two-photon microscopy. Microsc Res Tech 1999;47:163-4.

[19] Soeller C, Cannell MB. Two-photon microscopy: imaging in scattering samples and three-dimensionally resolved flash photolysis. Microsc Res Tech 1999;47:182-95.

[20] Denk W, Svoboda K. Photon upmanship: why multiphoton imaging is more than a gimmick. Neuron 1997;18:351-7.

[21] So PT, Dong CY, Masters BR, Berland KM. Two-photon excitation fluorescence microscopy. Annu Rev Biomed Eng 2000;2:399-429.

[22] Konig K, Riemann I. High-resolution multiphoton tomography of human skin with subcellular spatial resolution and picosecond time resolution. J Biomed Opt 2003;8:432-9.

[23] Masters BR, So PT, Buehler C, Barry N, Sutin JD, Mantulin WW. Mitigating thermal mechanical damage potential during two-photon dermal imaging. J Biomed Opt 2004;9:1265-70.

[24] Huzaira M, Rius F, Rajadhyaksha M, Anderson RR, González S. Topographic variations in normal skin, as viewed by in vivo reflectance confocal microscopy. J Invest Dermatol 2001;116:846-52.

[25] Yu B, Dong CY, So PT, Blankschtein D, Langer R. In vitro visualization and quantification of oleic acid induced changes in transdermal transport using two-photon fluorescence microscopy. J Invest Dermatol 2001;117:16-25.

[26] Kushner J, Kim D, So PT, Blankschtein D, Langer R. Dual-channel two-photon microscopy study of transdermal transport in skin treated with low-frequency ultrasound and a chemical enhancer. J Invest Dermatol 2007;127:2832-46.

[27] Johnson JC, Yan HQ, Schaller RD, Peterson PB, Yang PD, Saykally RJ. Near-field imaging of nonlinear optical mixing in single zinc oxide nanowires. Nano Lett 2002;2:279-83.

[28] Neumann U, Grunwald R, Griebner U, Stainmeyer G, Seeber W. Secondharmonic efficiency of ZnO nanolayers. Appl Phys Lett 2004;84:170-3.

[29] Noack V, Eychmuller A. Annealing of nanometer-sized zinc oxide particles. Chem Mater 2002;14:1411-7.

[30] Vanheusden K, Warren WL, Seager CH, Tallant DR, Voigt JA, Gnade BE. Mechanisms behind green photoluminescence in $\mathrm{ZnO}$ phosphor powders. J Appl Phys 1996;79:7983-90.

[31] Li Y, Cheng GS, Zhang LD. Fabrication of highly ordered ZnO nanowire arrays in anodic alumina membrane. J Mater Res 2000;15:2305-8.

[32] Hu JQ Li Q, Wong NB, Lee CS, Lee ST. Synthesis of uniform hexagonal prismatic ZnO whiskers. Chem Mater 2002;14:1216-9.

[33] Crossa SE, Innes B, Roberts MS, Tsuzuki T, Terry A, McCormick P. Human skin penetration of sunscreen nanoparticles: in-vitro assessment of a novel micronized zinc oxide formulation. Skin Pharmacol Physiol 2007;20:148-54. 